\title{
QCD Glueball Sum Rules Revisited
}

\author{
Hilmar Forkel \\ IFT, Universidade Estadual Paulista, Rua Pamplona, 145, 01405-900 São Paulo, SP, Brazil \\ Institut für Theoretische Physik, Universität Heidelberg, D-69120, Heidelberg, Germany
}

Received on 15 August, 2003.

\begin{abstract}
We discuss several key problems of conventional QCD glueball sum rules in the spin- 0 channels and show how they are overcome by nonperturbative Wilson coefficients. The nonperturbative contributions originate from direct instantons and, in the pseudoscalar channel, additionally from topological charge screening. The treatment of the direct-instanton sector is based on realistic instanton size distributions and renormalization at the operator scale. The resulting predictions for spin- 0 glueball properties as well as their implications for experimental glueball searches are discussed.
\end{abstract}

\section{Introduction}

The gluonium sector of QCD has remained complex and intriguing for almost four decades [1]. Its "exotic" nature partially reflects itself in several longstanding problems which the QCD sum rule approach faces in the spin0 glueball channels [2]. In the scalar $\left(0^{++}\right)$glueball correlator, in particular, the departure from asymptotic freedom sets in at surprisingly small distances [3]. Unusually strong, nonperturbative contributions to the Wilson coefficients are then required to provide stability for the $0^{++}$ glueball sum rules (mainly the one including a large subtraction constant) and to reconcile them with an underlying low-energy theorem (see below) [4]. While instantons [5] seemed to be a likely candidate for such contributions early on [6], their implementation into the short-distance expansion (i.e. the OPE) had to await better knowledge of the instanton size distribution $[5,7]$. The instanton-improved OPE (IOPE) and the analysis of the corresponding Borel sum rules [4] then showed that direct (i.e. small-scale) instantons solve two key problems in the scalar glueball channel: they render for the first time all Borel-moment sum rules individually and mutually consistent. A subsequent analysis of the related Gaussian sum rules [8] confirmed some of these findings and investigated alternative parametrizations of the phenomenological side. However, the previous implementations of direct instanton contributions, including those in the $0^{++}$glueball channel, relied on approximations which may cause artefacts in the sum-rule results. We will therefore outline a more thorough and systematic treatment here. Moreover, while direct-instanton contributions resolve longstanding shortcomings in the scalar glueball sum rules, their straightforward implementation into the $0^{-+}$sum rules seems, at first, to create new problems [9]. These problems have an appealing solution, however, due to topological charge screening [10], as we will discuss below.

\section{Correlators and sum rules}

Our discussion will be based on the correlations functions of the scalar $\left(0^{++}\right)$and pseudoscalar $\left(0^{-+}\right)$glueball channels, defined as

$$
\Pi_{G}(x)=\left\langle 0\left|T O_{G}(x) O_{G}(0)\right| 0\right\rangle
$$

where $O_{G}$ with $G \in\{S, P\}$ are the standard gluonic interpolating fields (with lowest mass dimension)

$$
\begin{aligned}
& O_{S}(x)=\alpha_{s} G_{\mu \nu}^{a}(x) G^{a \mu \nu}(x), \\
& O_{P}(x)=\alpha_{s} G_{\mu \nu}^{a}(x) \tilde{G}^{a \mu \nu}(x) .
\end{aligned}
$$

The correponding Fourier transforms are

$$
\Pi_{G}\left(-q^{2}\right)=i \int d^{4} x e^{i q x}\left\langle 0\left|T O_{G}(x) O_{G}(0)\right| 0\right\rangle .
$$

The zero-momentum limits of these correlators are governed by low-energy theorems:

$$
\Pi_{S}\left(q^{2}=0\right)=\frac{32 \pi}{b_{0}}\left\langle\alpha G^{2}\right\rangle
$$

in the scalar channel [11] and (with three light flavors and $\left.m_{u, d} \ll m_{s}\right)$

$$
\Pi_{P}\left(q^{2}=0\right)=(8 \pi)^{2} \frac{m_{u} m_{d}}{m_{u}+m_{d}}\langle\bar{q} q\rangle
$$

(which vanishes in the chiral limit) in the pseudoscalar one [12]. Consistency with the low-energy theorems is a stringent requirement for the sum rules which can be met only by nonperturbative short-distance physics in the IOPE $[4,10]$.

The direct-instanton contributions to the glueball correlators show a pronounced and robust channel dependence pattern which originates from the (Minkowski) (anti-) selfduality of the (anti-) instanton's field strength,

$$
G_{\mu \nu}^{(I, \bar{I})}= \pm i \tilde{G}_{\mu \nu}^{(I, \bar{I})} \equiv \frac{i}{2} \varepsilon_{\mu \nu \rho \sigma} G_{\rho \sigma}^{(I, \bar{I})} .
$$

Self-duality implies that the direct-instanton contributions to the $0^{-+}$glueball correlator are equal in size and opposite in sign to those in the $0^{++}$glueball correlator. Moreover, the energy-momentum tensor of self-dual fields vanishes, and consequently their contributions to the tensor glueball 
correlator (based on the interpolator $O_{T}(x)=\Theta_{\mu \nu}^{a}(x)$ ). Since the instanton contributions to the scalar glueball correlator turn out to be strongly attractive, they must be equally strongly repulsive in the pseudoscalar channel.

In order to make contact with the hadronic information contained in the glueball correlators, we now turn to the dispersive representation

$$
\Pi_{G}\left(Q^{2}\right)=\frac{1}{\pi} \int_{0}^{\infty} d s \frac{\operatorname{Im} \Pi_{G}(-s)}{s+Q^{2}}
$$

where the necessary number of subtractions is implied but not written explicitly. The standard sum-rule description of the spectral functions contains one or two resonance poles (in zero-width approximation) and the local-duality continuum, i.e.

$$
\operatorname{Im} \Pi_{G}^{(p h)}(s)=\operatorname{Im} \Pi_{G}^{(p o l e)}(s)+\operatorname{Im} \Pi_{G}^{(\text {cont })}(s)
$$

with

$$
\begin{aligned}
& \operatorname{Im} \Pi^{(\text {pole })}(s)=\pi \sum_{i=1}^{2} f_{G i}^{2} m_{G i}^{4} \delta\left(s-m_{G i}^{2}\right), \\
& \operatorname{Im} \Pi_{G}^{(\text {cont })}(s)=\theta\left(s-s_{0}\right) \operatorname{Im} \Pi_{G}^{(I O P E)}(s) .
\end{aligned}
$$

The continuum representation is obtained from the discontinuities of the IOPE and covers the invariant-mass region "dual" to higher-lying resonances and multi-hadron continuum, starting at an effective threshold $s_{0}$.

In order to write down QCD sum rules, the correlators weighted with powers of $-Q^{2}$ - are Borel-transformed,

$$
\mathcal{L}_{G, k}^{(X)}(\tau)=\hat{B}\left[\left(-Q^{2}\right)^{k} \Pi_{G}^{(X)}\left(Q^{2}\right)\right](\tau), \quad k \geq-1 .
$$

The hadronic parameters $m_{G i}, f_{G i}, s_{0}$ are then determined by matching the moments $\mathcal{L}_{G, k}^{(p h)}\left(\tau ; m_{G i}, f_{G i}, s_{0}\right)$ in the fiducial $\tau$-region to their counterparts $\mathcal{L}_{G, k}^{(I O P E)}$ which will be determined below. The ensuing IOPE sum rules are conveniently written in terms of the continuum-subtracted Borel moments $\mathcal{R}_{G, k}$ as

$$
\begin{aligned}
\mathcal{R}_{G, k}\left(\tau ; s_{0}\right) & \equiv \frac{1}{\pi} \int_{0}^{s_{0}} d s s^{k} \operatorname{Im} \Pi_{G}^{(I O P E)}(s) e^{-s \tau} \\
& =\sum_{i=1}^{2} f_{G i}^{2} m_{G i}^{4+2 k} e^{-m_{G i}^{2} \tau}-\delta_{k,-1} \Pi_{G}^{(p h)}(0)
\end{aligned}
$$

which isolates the pole contributions of interest (and the important subtraction term for $k=-1$ ) on the RHS.

\section{IOPE}

Our theoretical framework for calculating the correlator amplitudes at short distances is the instanton-improved operator product expansion (IOPE). The general IOPE expression for the glueball correlators at large, spacelike momenta $Q^{2} \equiv-q^{2} \gg \Lambda_{Q C D}$,

$$
\Pi_{G}\left(Q^{2}\right)=\sum_{D=0,4, \ldots} \tilde{C}_{D}^{(G)}\left(Q^{2} ; \mu\right)\left\langle\hat{O}_{D}\right\rangle_{\mu}
$$

exhibits the characteristic factorization into contributions from "hard" field modes (with momenta $|k|>\mu$ ) in the Wilson coefficients $\tilde{C}_{D}\left(Q^{2}\right)$ and contributions from "soft" field modes (with $|k| \leq \mu$ ) in the vacuum expectation values (condensates") of the operators $\hat{O}_{D}$ of dimension $D$.

The perturbative Wilson coefficients, which generate the conventional OPE, can be found in Refs. $[8,10]$ for the $0^{++}$ and in Refs. $[9,10,13]$ for the $0^{-+}$channel. In the following we will focus on the nonperturbative contributions due to direct instantons and topological-charge screening. The unit-operator coefficient $\tilde{C}_{0}^{(G)}$ of the IOPE receives the dominant direct-instanton contribution [10],

$$
\Pi_{G}^{(I+\bar{I})}\left(x^{2}\right)=\frac{2^{8} 3}{7} \int d \rho n(\rho) \frac{1}{\rho^{4}}{ }_{2} F_{1}\left(4,6, \frac{9}{2},-\frac{x^{2}}{4 \rho^{2}}\right) .
$$

From its Fourier transform one finds the corresponding Borel moments [4] $\left(x=\rho^{2} / 2 \tau\right)$

$$
\begin{aligned}
\mathcal{L}_{-1}^{(I+\bar{I})}(\tau) & =-2^{6} \pi^{2} \int d \rho n(\rho) x^{2} e^{-x} \\
& \times\left[(1+x) K_{0}(x)+\left(2+x+\frac{2}{x}\right) K_{1}(x)\right]
\end{aligned}
$$

(which, for $\tau \rightarrow \infty$, approaches the subtraction term $\left.-\delta_{k,-1} \Pi_{G}^{(I+\bar{I})}(0)\right)$ and

$$
\mathcal{L}_{k+1}^{(I+\bar{I})}(\tau)=\frac{-\partial}{\partial \tau} \mathcal{L}_{k}^{(I+\bar{I})}(\tau) \quad(\text { for } k \geq-1) .
$$

From the imginary part [4]

$$
\operatorname{Im} \Pi_{S}^{(I+\bar{I})}(-s)=-2^{4} \pi^{4} \int d \rho n(\rho) \rho^{4} s^{2} J_{2}(\sqrt{s} \rho) Y_{2}(\sqrt{s} \rho)
$$

at timelike momenta one then has

$$
\begin{aligned}
\mathcal{R}_{k}^{(I+\bar{I})}(\tau) & =-2^{7} \pi^{2} \delta_{k,-1} \int d \rho n(\rho)-2^{4} \pi^{3} \int d \rho \\
& \times n(\rho) \rho^{4} \int_{0}^{s_{0}} d s s^{k+2} J_{2}(\sqrt{s} \rho) Y_{2}(\sqrt{s} \rho) e^{-s \tau} .
\end{aligned}
$$

The only input for the evaluation of these contribution is the (anti-) instanton distributions $n(\rho)$. The Wilson coefficients are primarily sensitive to the qualitative behavior of $n(\rho)$ and two characteristic scales, the leading moments

$$
\bar{n}=\int d \rho n(\rho), \quad \bar{\rho}=\frac{1}{\bar{n}} \int d \rho \rho n(\rho) .
$$

All previous direct-instanton calculations have relied on the oversimplified "spike" approximation $n(\rho)=\bar{n} \delta(\rho-\bar{\rho})$. Additional features of $n(\rho)$ (shape, small- and large- $\rho$ behavior) are by now well enough established, however, to make more realistic parametrizations possible. Artefacts of the spike approximation (e.g. oscillations in the time-like region) are thereby avoided. Hence we introduce ansätze for $n(\rho)$ which are uniquely determined by $\bar{n}, \bar{\rho}$ and their 
small- and large- $\rho$ behavior [10]. The most realistic one is the Gaussian-tail distribution (for $N_{c}=N_{f}=3$ )

$$
n_{g}(\rho)=\frac{2^{18}}{3^{6} \pi^{3}} \frac{\bar{n}}{\bar{\rho}}\left(\frac{\rho}{\bar{\rho}}\right)^{4} \exp \left(-\frac{2^{6}}{3^{2} \pi} \frac{\rho^{2}}{\bar{\rho}^{2}}\right)
$$

which starts as $\rho^{4}$ at small $\rho$, as required by instantonbackground perturbation theory.

Realistic instanton size distributions have an additional benefit: they allow to renormalize the direct-instanton contributions at the operator scale $\mu$. Although the neglect of renormalization is common practice ("pragmatic OPE") for the perturbative Wilson coefficients, it is completely untested in the nonperturbative sector. Since instantonassociated flutuations carry a momentum scale $k \sim \rho^{-1}$, we implement $\mu$ (gauge invariantly) by excluding contributions from instantons with size $\rho>\mu^{-1}$, i.e. by replacing

$$
n(\rho) \rightarrow \tilde{n}_{\mu}(\rho) \equiv \theta_{\beta}\left(\rho-\mu^{-1}\right) n(\rho)
$$

where the "soft" step function $\theta_{\beta}$ can be choosen, e.g. as a Fermi distribution with "diffuseness" $\beta$. We find the instanton-induced Wilson coefficients to be relatively weakly $\mu$-dependent for $\mu<\bar{\rho}^{-1}$ where the large- $\rho$ suppression from $n(\rho)$ at $\rho<\mu^{-1}$ has already set in. This is reassuring since the compensating $\mu$-dependence of the condensates is equally weak. The standard spike distribution (with $\bar{\rho}<\mu^{-1}$ ) misses the reduction of the total instanton density active in the Wilson coefficients,

$$
\bar{n}=\int_{0}^{\infty} d \rho n(\rho) \rightarrow \int_{0}^{\infty} d \rho \tilde{n}_{\mu}(\rho) \equiv \bar{n}_{\text {direct }} .
$$

Comparison of the imaginary part (18) from $n_{g}(\rho)$ to that obtained from the spike distribution shows that the finite-width distribution turns an oscillating rise at large $s$ into a strong decay $\propto s^{-5 / 2}$. Moreover, for $s_{0} \gtrsim 2-4$ $\mathrm{GeV}^{2}$ (and $\tau \gtrsim 0.2 \mathrm{GeV}^{-1}$ ) the finite-width distribution increasingly reduces the size of the instanton contributions. Therefore, they will be smaller in the pseudoscalar sum rule (where $s_{0}$ is typically about a factor of two larger) than in the scalar sum rule.

\section{Topological charge screening}

Due to the sign change originating from (Minkowski) selfduality, the instanton-induced attraction in the $0^{++}$glueball correlator turns into repulsion for the $0^{-+}$correlator. The strength of the direct-instanton contributions explains why their inclusion in the pseudoscalar IOPE makes the signal for the pseudoscalar glueball and the compulsory spectral positivity disappear [9]. Additional and equally important contributions to the Wilson coefficients must therefore still be missing. These contributions must affect mostly the pseudoscalar IOPE since the scalar sum rules are consistent and stable without them.

Such a strong channel selectivity is indeed possible since the pseudoscalar correlator is proportional to the topological charge density correlator and thus maximally sensitive to instanton - antiinstanton correlations. Correlations of this sort are created by the attractive (repulsive) $\eta^{\prime}$-meson exchange forces between opposite-sign (equal-sign) topological charges in the QCD vacuum and lead to their Debye screening [14]. The large $\eta^{\prime}$ mass is the corresponding "screening mass", and $\lambda_{D} \sim m_{\eta^{\prime}}^{-1} \sim 0.2 \mathrm{fm}$ the (small!) screening length. Since $m_{\eta^{\prime}}>\mu$, the screening correlations contribute to the Wilson coefficients of the pseudoscalar glueball correlator.

The screening corrections can be obtained from the coupling of the $\eta_{0}$ meson (the flavor-singlet part of the $\eta^{\prime}$ ) to the topological charge density in the vacuum medium (approximated for simplicity as concentrated in pointlike instantons) which is dictated by the axial anomaly [15] and governed by the effective lagrangian [14]

$\mathcal{L}=\frac{1}{2}\left(\partial \eta_{0}\right)^{2}+\frac{1}{2} m_{0}^{2} \eta_{0}^{2}-\xi \cos \left(\theta(x)+\frac{\sqrt{2 N_{f}}}{f_{\eta^{\prime}}} \eta_{0}(x)\right)$.

Here $\xi$ is the overall topological charge density ( $=\bar{n}$ for instantons) and $\theta(x)$ is a source for the topological charge density $Q(x)$. Taking two derivatives of the corresponding generating functional with respect to $\theta$ leads (for small $\eta_{0}$ amplitudes) to the topological charge correlator

$$
\begin{aligned}
\langle Q(x) Q(0)\rangle & =\Pi_{P}(x) /(8 \pi)^{2} \\
& \simeq-2 \xi \delta^{4}(x)-8 N_{f} \frac{\xi^{2}}{f_{\eta^{\prime}}^{2}}\left\langle\eta_{0}(x) \eta_{0}(0)\right\rangle .
\end{aligned}
$$

The first term is just the pointlike-instanton approximation of the direct-instanton contribution evaluated above. The second one is the screening correction which, after correcting for $\eta_{0}-\eta_{8}$ mixing, adds the contribution

$$
\begin{aligned}
\mathcal{R}_{P, k}^{(s c r)}(\tau) & =-\delta_{k,-1}\left(\frac{F_{\eta^{\prime}}^{2}}{m_{\eta^{\prime}}^{2}}+\frac{F_{\eta}^{2}}{m_{\eta}^{2}}\right) \\
& +F_{\eta^{\prime}}^{2} m_{\eta^{\prime}}^{2 k} e^{-m_{\eta^{\prime}}^{2} \tau}+F_{\eta}^{2} m_{\eta}^{2 k} e^{-m_{\eta}^{2} \tau}
\end{aligned}
$$

to the pseudoscalar IOPE moments. The quantitative analysis of the associated sum rules reveals that the screening contributions indeed resolve the detrimental problems mentioned above: positivity of the spectral function is restored, all sum rules (including an $\eta^{\prime}$ pole on the RHS) are stable, and they provide a clear signal for the pseudoscalar glueball (for more details see Ref. [10]).

\section{Glueball predictions}

On the basis of the improved and extended IOPE we have performed a comprehensive numerical analysis of eight Borel sum rules in both spin-0 glueball channels. The results reveal a rather diverse pattern of glueball properties. In the scalar channel, the improved treatment of the direct-instanton sector reduces our earlier (spikedistribution based) result for the $0^{++}$glueball mass by about $20 \%$, to $m_{S}=1.25 \pm 0.2 \mathrm{GeV}$. This is somewhat smaller than the quenched lattice results [16] which are, however, 
expected to be reduced by light-quark effects and quarkonium admixtures.

Our mass prediction is consistent with the broad glueball state found in a recent $K$-matrix analysis which includes the new states recently identified in the Crystal Barrel data [17]. The systematics in our results from different Borel-moment sum rules indicates a rather large width of the scalar glueball, $\Gamma_{S} \gtrsim 0.3 \mathrm{GeV}$. Our prediction for the glueball decay constant, $f_{S}=1.05 \pm 0.1 \mathrm{GeV}$, is several times larger than the value obtained when ignoring the nonperturbative Wilson coefficients. This result implies an exceptionally small glueball size and at least partially explains the strong attraction between gluons in the scalar channel as instanton-induced. Furthermore, our prediction for $f_{S}$ implies substantially larger partial widths of radiative $J / \psi$ and $\Upsilon$ decays into scalar glueballs and is therefore important for experimental glueball searches, in particular for the interpretation of the recent CLEO [18] and forthcoming CLEO-III data on $\Upsilon \rightarrow \gamma f_{0}$ and other decay branches.

In the pseudoscalar glueball channel, the hard nonperturbative contributions modify qualitative features of the $0^{-+}$Borel moments to which the matching analysis is particularly sensitive, and they are vital for achieving consistency among all moment sum rules and with the axial anomaly. Our mass prediction $m_{P}=2.2 \pm 1.5 \mathrm{GeV}$ for the pseudoscalar glueball lies inside the range obtained from quenched and unquenched lattice data. The coupling $f_{P}=0.6 \pm 0.2 \mathrm{GeV}$ is again enhanced by the nonperturbative Wilson coefficients, but less strongly than in the scalar channel. The consequently larger partial width of radiative quarkonium decays into pseudoscalar glueballs and the enhanced $\gamma \gamma \rightarrow G_{P} \pi^{0}$ cross section at high momentum transfers will be relevant for the experimental identification of the lowest-lying $0^{-+}$glueball and help in measuring its properties.

Our nonperturbative IOPE, including the topological short-distance physics described above, should also be useful for the calculation of other spin- 0 glueball properties. Quantitative estimates of the already mentioned production rates in gluon-rich channels (including $J / \psi$ and $\Upsilon$ decays) and characteristic glueball decay properties and signatures, including $\gamma \gamma$ couplings, OZI suppression and branching fractions incompatible with $q \bar{q}$ decay, would be particularly interesting.

This work was supported by FAPESP.

\section{References}

[1] M. Gell-Mann, Acta Phys. Aust. Suppl. 9, 733 (1972); H. Fritzsch and M. Gell-Mann, 16th Int. Conf. High-Energy Phys., Chicago, Vol. 2, 135 (1972).

[2] S. Narison, Nucl. Phys. B509, 312 (1998) and references therein.

[3] V.A. Novikov, M.A. Shifman, A.I. Vainsthein, and V.I. Zakharov, Nucl. Phys. B191, 301 (1981).

[4] H. Forkel, Phys. Rev. D 64, 34015 (2001); hep-ph/0005004.

[5] T. Schaefer and E.V. Shuryak, Rev. Mod. Phys. 70, 323 (1998). For an elementary introduction see H. Forkel, A Primer on Instantons in $Q C D$, hep-ph/0009136.

[6] V.A. Novikov, M.A. Shifman, A.I. Vainsthein, and V.I. Zakharov, Nucl. Phys. B165, 67 (1980).

[7] A. Ringwald and F. Schremmp, Phys. Lett. B459, 249 (1999).

[8] D. Harnett and T.G. Steele, Nucl. Phys. A695, 205 (2001).

[9] A. Zhang and T.G. Steele, hep-ph/0304208.

[10] H. Forkel, Direct instantons, topological charge screening and QCD glueball sum rules, preprint IFT-P.039/2003, hep$\mathrm{ph} / 0312049$.

[11] V.A. Novikov, M.A. Shifman, A.I. Vainshtein and V.I. Zakharov, Nucl. Phys. B191, 301 (1981).

[12] H. Leutwyler and A. Smilga, Phys. Rev. D 46, 5607(1992).

[13] V.A. Novikov, M.A. Shifman, A.I. Vainsthein, and V.I. Zakharov, Phys. Lett. B 86, 347 (1979).

[14] N.J. Dowrick and N.A. McDougall, Phys. Lett. B 285, 269 (1992); H. Kikuchi and J. Wudka, Phys. Lett. B 284, 111 (1992).

[15] P. Di Vecchia and G. Veneziano, Nucl. Phys. B 171, 253 (1980).

[16] W. Lee and D. Weingarten, Phys. Rev. D 61, 014015 (2000) and references therein; C. Morningstar and M. Peardon, Phys. Rev. D 60, 034509 (1999).

[17] V.V. Anisovich, hep-ph/0310165.

[18] CLEO Collaboration, A. Anastassov et al., Phys. Rev. Lett. 82, 286 (1999); S. Richichi et al., ibid. 87, 141801 (2001); G. Masek et al., Phys. Rev. D 65, 072002 (2002). 